\title{
The Add-on Effects of Danhong Injection Among Patients With Ischemic Stroke Receiving Western Medicines: a Systematic Review and Meta-analysis
}

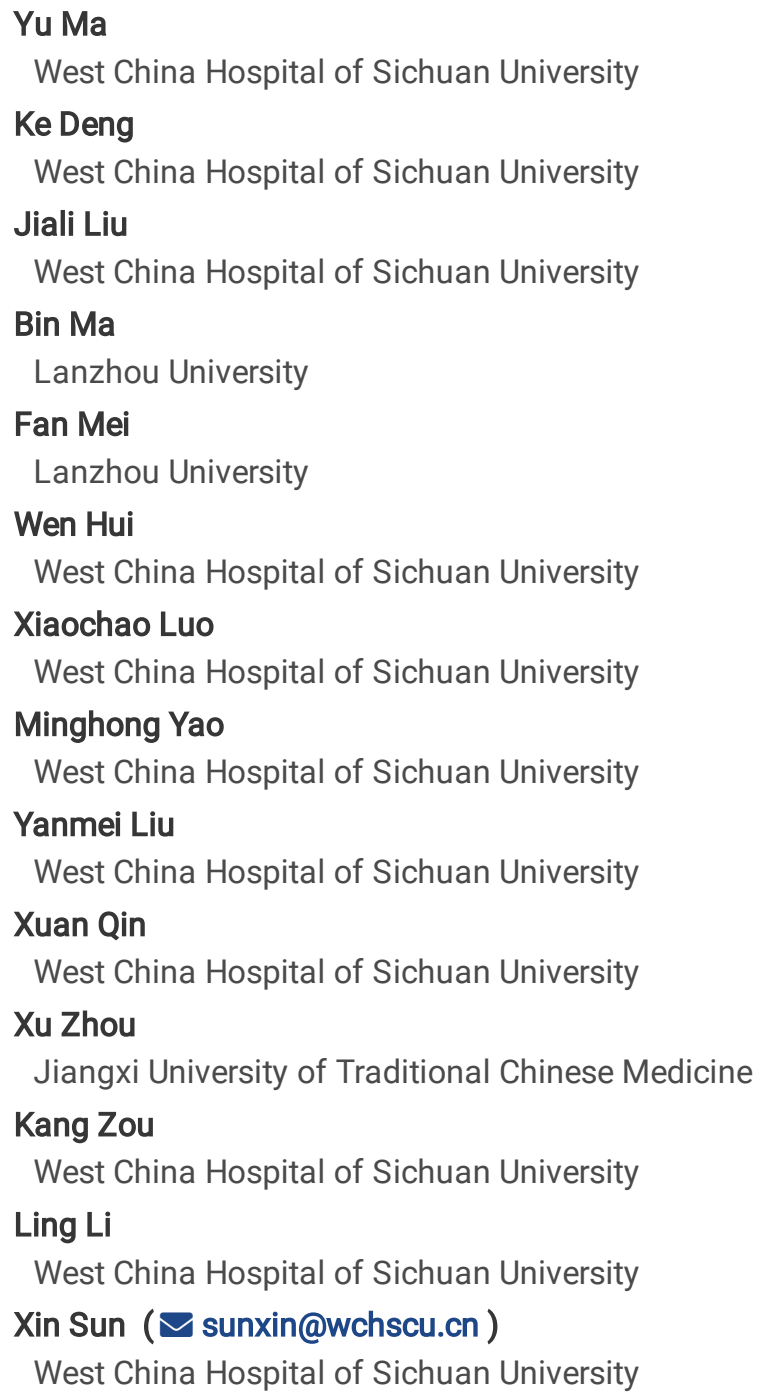

\section{Research Article}

Keywords: Danhong Injection, Ischemic Stroke, Meta-analysis, Systematic review

Posted Date: February 7th, 2022

DOI: https://doi.org/10.21203/rs.3.rs-1305884/v1

License: (c) (1) This work is licensed under a Creative Commons Attribution 4.0 International License. Read Full License 


\section{Abstract}

Background: Danhong injection is widely used for treating ischemic stroke in China. However, its effects on ischemic stroke patients when adding to western medicines (i.e., the add-on effect) were not well established.

Methods: We searched PubMed, Embase, Cochrane Central Register of Controlled Trials (CENTRAL) and three Chinese databases from inception to 20 July 2020 to identify randomized clinical trials (RCTs) that assessed the effects of Danhong injection as an add-on therapy in patients with ischemic stroke. Pairs of trained reviewers independently screened for eligible studies, assessed risk of bias, and extracted data. The outcomes were National Institutes of Health Stroke Scale Score (NIHSS), Barthel index, activities of daily living $(A D L)$, total cholesterol and homocysteine (Hcy).

Results: 67 RCTs of 6594 patients with varying risk of bias were included. Compared with western medicine alone, the addition of Danhong injection to western medicine significantly lowered NIHSS score (45 RCTs with 4565 patients; MD -4.21, 95\% Cl -4.96区-3.46), total cholesterol (10 trials with 1019 patients; MD -1.14 mmol/L, 95\% Cl-1.57ه-0.72) and Hcy (4 trials with 392 patients; MD -3.54 $\mu \mathrm{mol} / \mathrm{L}, 95 \% \mathrm{Cl}-4.38 \rrbracket-2.07$ ). The addition of Danhong also increased Barthel index (14 trials with 1270 patients; $\mathrm{MD} 8.71,95 \% \mathrm{Cl} 3.68$ ه13.74) and ADL (12 trials with 1114 patients; MD 14.48, 95\% Cl 9.04囚19.92) scores. Subgroup analyses showed differential effects in average cerebral blood flow rate by mean age of patients (<60 years: MD $0.74 \mathrm{~cm} / \mathrm{s}, 95 \% \mathrm{Cl} 0.29 \otimes 1.19 ; \geq 60$ years: MD $4.09 \mathrm{~cm} / \mathrm{s}, 95 \%$ $\mathrm{Cl} 2.02 \varangle 6.16$; interaction $\mathrm{P}=0.002$ ) and NIHSS score by type of baseline western medicines (interaction $\mathrm{P}<0.00001$ ).

Conclusions: The addition of Danhong injection to western medicine may improve neurological function, self-care ability and blood lipids of ischemic stroke patients. Furthermore, patients with different age and receiving different baseline western medicines may have different benefits in hemodynamic status and neurological function, respectively.

\section{Background}

Ischemic stroke, defined as all thromboembolic and atherosclerotic events resulting in compromised blood flow to cerebral tissue and subsequent infarction[1], is a leading cause of death and long-term disability worldwide. Current recommendations for patients with ischemic stroke include antiplatelet, anticoagulants, volume expansion, vasodilators, neuroprotective agents, intravenous thrombolysis and mechanical thrombectomy[2]. In spite of these, patients with ischemic stroke continue to suffer from high risk of disability and recurrence, and add-on treatments are often necessary [3].

Danhong injection is a Chinese patent medicine extracted from Salviae Miltiorrhizae (Danshen in Chinese) and Flos Carthami (Honghua in Chinese), which improve microcirculation, prevent platelet aggregation, decrease plasma viscosity and boost the activity of fibrinogen dissolved. Danhong injection has been widely used for cardiovascular and cerebrovascular diseases [4,5]. Evidence from randomized clinical trials (RCTs) with small sample sizes showed that the combination of Danhong injection and western medicine was effective in improving total efficiency and hemodynamic outcomes among ischemic stroke patients[6-8]. Three systematic reviews also found that, compared with other Chinese herbal injections (e.g., Shuxuening injection), Danhong injection appeared to be beneficial in total clinical effectiveness rate and neurologic impairment [9-11]. However, these reviews included a relatively small number of RCTs, and were limited with improper comparisons, inappropriate outcome selection and analysis, as a result of which the resulting findings and recommendations were not definitive.

Most importantly, Danhong injection is routinely used as an add-on therapy to patients with ischemic stroke receiving western medicine; its add-on effects on ischemic stroke patients still need further systematic assessment to inform current practice. Therefore, we conducted this systematic review and meta-analyses which included a significantly larger number of newly published RCTs.

\section{Methods}

We followed Preferred Reporting Items for Systematic Reviews and Meta-analyses (PRISMA) statement[12] to conduct and report this systematic review and meta-analysis. This protocol was registered with the International Prospective Register of Systematic Reviews (CRD42022298628).

\section{Eligibility criteria}


We included randomized controlled trials (RCTs) that compared efficacy and safety of Danhong injection combined with western medicine therapy versus western medicine alone in patients with ischemic stroke. There was no limitation on the dosages and courses of treatment. Eligible studies explicitly reported outcomes data on National Institute of Health Stroke Scale (NIHSS), Fugl-Meyer Assessment, Barthel index, activities of daily living (ADL), intima-media thickness (IMT), cerebral blood flow, average cerebral blood flow rate, total cholesterol, triglycerides, high-density lipoprotein (HDL), low-density lipoprotein (LDL), homocysteine (Hcy), D-dimer and adverse drug reactions (ADRs)/adverse drug events (ADEs). We excluded RCTs that involved other Chinese herbal medicine.

\section{Literature search}

We searched PubMed, Embase, Cochrane Central Register of Controlled Trials (CENTRAL), China National Knowledge Infrastructure Database (CNKI), Wanfang database and Chinese Scientific Journal Database to identify relevant studies from inception to 20 July, 2020. The subject terms (e.g., MeSH terms) and free-text words were used to search for potentially eligible studies, and language restriction was imposed in English and Chinese. The specific retrieval strategy was provided in Appendix 1.

\section{Study process}

Two reviewers (Yu Ma and Ke Deng) independently screened titles/abstracts and full texts for eligibility, assessed risk of bias, and collected data from each eligible study using a prespecified form. Reviewers dealt with discrepancies through discussion or, if required, adjudication by a third researcher (Ling Li).

\section{Risk of bias assessment}

We used the modified Cochrane Risk of Bias Assessment Tool to assess risk of bias of included RCTs[13]. The items contained random sequence generation (selection bias), allocation concealment (selection bias), blinding of patients and personnel (performance bias), blinding of outcome assessment (detection bias), incomplete outcome data (attrition bias), selective outcome reporting (reporting bias), and other sources of bias. The judgment for each item included low/high/unclear risk of bias.

\section{Data extraction}

We collected the following information from included RCTs: general study characteristics (first author, year of publication, total number of patients randomized); patients' characteristics (age, sex); intervention and control characteristics (medications used across groups (baseline treatment), details of Danhong injection treatment and control group (dose, type of western medicines used), and course of treatment); and outcomes data (mean and standard deviation of continuous variables, and number of patients occurring adverse drug reactions/adverse drug events in each group).

\section{Statistical Analysis}

The mean difference (MD) was calculated for continuous variables; effects of each outcome were estimated with $95 \%$ confidence intervals $(95 \% \mathrm{Cls})$. We used the Cochran's Chi-squared test and the ${ }^{2}$ statistic to examine statistical heterogeneity among RCTs. Metaanalysis was calculated by random-effects model.

We explored sources of heterogeneity with three prespecified subgroup hypotheses: length of treatment ( $\leq 2$ vs $>2$ weeks; larger effect in trials with longer treatment), mean age of patients ( $<60 \mathrm{vs} \geq 60$ years old; larger effect in trials with younger patients) and types of baseline western medicine treatment (conventional therapy vs intravenous thrombolysis vs anti-platelet aggregation vs dilation of blood vessels vs statins vs neuroprotective agents vs other therapies).

In addition, we used the funnel plot and Egger's test for examining publication bias [14]. We also used the Duval and Tweedie trim-andfill method for exploring the influence of a single trial on publication bias [15].

\section{Results}

As shown in Figure 1, our search retrieved 3080 citations during the initial detection by the search strategy. After screening titles and abstracts, 671 reports were included for full further text screening. Sixty-seven RCTs were eligible for meta-analysis ultimately.

These 67 RCTs $[6-8,16-79]$ were comprised of 3293 patients in the combination treatment group and 3301 patients in the control group. The mean age of included patients ranged from 42.2 to 71.5 years, and the sample size ranged from 40 to 300 patients. All 
RCTs were conducted among Chinese populations in China. Patients in the combination treatment group received Danhong injection 10-40 ml once or twice daily (Table 1). 
Table 1

Baseline characteristics of included randomized controlled trials

\begin{tabular}{|c|c|c|c|c|c|c|c|c|c|}
\hline \multirow[t]{2}{*}{$\begin{array}{l}\text { Author } \\
\text { (year) }\end{array}$} & \multirow{2}{*}{$\begin{array}{l}\text { Phase } \\
\text { of } \\
\text { disease }\end{array}$} & \multirow{2}{*}{$\begin{array}{l}\text { Sample } \\
\text { size, } \\
\text { EG/CG }\end{array}$} & \multicolumn{2}{|c|}{$\begin{array}{l}\text { Mean age } \\
\text { (year) }\end{array}$} & \multicolumn{2}{|l|}{ Interventions } & \multirow{2}{*}{$\begin{array}{l}\text { Dosages } \\
\text { of } \\
\text { Danhong } \\
\text { Injection }\end{array}$} & & \multirow[t]{2}{*}{ Outcomes } \\
\hline & & & EG & CG & EG & CG & & & \\
\hline $\begin{array}{l}\text { Cao } \\
2020\end{array}$ & $\begin{array}{l}\text { Acute } \\
\text { phase }\end{array}$ & $49 / 49$ & 63.11 & 62.47 & rt-PA, DHI & rt-PA & $\begin{array}{l}\text { Once } \\
\text { daily, } \\
20 \mathrm{ml}\end{array}$ & $14 d$ & $\begin{array}{l}(1)(12) \\
(14)\end{array}$ \\
\hline $\begin{array}{l}\text { Cheng } \\
2019\end{array}$ & NR & $20 / 20$ & 58.40 & 56.10 & Edaravone, DHI & Edaravone & $\begin{array}{l}\text { Once } \\
\text { daily, } \\
30 \mathrm{ml}\end{array}$ & $14 d$ & (14) \\
\hline $\begin{array}{l}\text { Zhang } \\
2019\end{array}$ & NR & $55 / 55$ & 53.80 & 53.20 & Clopidogrel, DHI & Clopidogrel & $\begin{array}{l}\text { Once } \\
\text { daily, } \\
30 \mathrm{mg}\end{array}$ & $28 d$ & $(14)$ \\
\hline $\begin{array}{l}\text { Deng } \\
2019\end{array}$ & NR & $50 / 50$ & 63.77 & 63.28 & Edaravone, DHI & Edaravone & $\begin{array}{l}\text { Once or } \\
\text { twice } \\
\text { daily, 20- } \\
40 \mathrm{ml}\end{array}$ & $14 d$ & (1) \\
\hline Li 2017 & $\begin{array}{l}\text { Acute } \\
\text { phase }\end{array}$ & $40 / 40$ & 66.50 & 66.50 & Edaravone, DHI & Edaravone & $\begin{array}{l}\text { Once } \\
\text { daily, } \\
30 \mathrm{ml}\end{array}$ & $14 d$ & $\begin{array}{l}(1)(4) \\
(14)\end{array}$ \\
\hline Li 2017 & $\begin{array}{l}\text { Acute } \\
\text { phase }\end{array}$ & $30 / 30$ & 61.48 & 62.03 & $\begin{array}{l}\text { Ozagrel Sodium, } \\
\text { DHI }\end{array}$ & Ozagrel Sodium & $\begin{array}{l}\text { Once } \\
\text { daily, } \\
40 \mathrm{ml}\end{array}$ & $14 d$ & $\begin{array}{l}(1)(8)(9) \\
10)(11)\end{array}$ \\
\hline $\begin{array}{l}\text { Wang } \\
2016\end{array}$ & $\begin{array}{l}\text { Acute } \\
\text { phase }\end{array}$ & $35 / 37$ & NR & NR & Aspirin, DHI & Aspirin & $\begin{array}{l}\text { Once } \\
\text { daily, } \\
40 \mathrm{ml}\end{array}$ & $14 d$ & (3) (14) \\
\hline $\begin{array}{l}\text { Zhang } \\
2015\end{array}$ & $\begin{array}{l}\text { Acute } \\
\text { phase }\end{array}$ & $50 / 50$ & 58.60 & 60.30 & Vinpocetine, DHI & Vinpocetine & $\begin{array}{l}\text { Once } \\
\text { daily, } \\
30 \mathrm{ml}\end{array}$ & $14 d$ & (1) \\
\hline $\begin{array}{l}\text { Yin } \\
2014\end{array}$ & $\begin{array}{l}\text { Acute } \\
\text { phase }\end{array}$ & $60 / 60$ & 52.80 & 53.10 & $\begin{array}{l}\text { Hyperbaric Oxygen, } \\
\text { DHI }\end{array}$ & Hyperbaric Oxygen & $\begin{array}{l}\text { Twice } \\
\text { daily, } \\
20 \mathrm{ml}\end{array}$ & $56 d$ & (1) \\
\hline $\begin{array}{l}\text { Shen } \\
2012\end{array}$ & $\begin{array}{l}\text { Acute } \\
\text { phase }\end{array}$ & $36 / 36$ & NR & NR & Edaravone, DHI & Edaravone & $\begin{array}{l}\text { Once } \\
\text { daily, } \\
30 \mathrm{ml}\end{array}$ & $14 d$ & $\begin{array}{l}(1)(4) \\
14)^{(4)}\end{array}$ \\
\hline $\begin{array}{l}\text { Zhuang } \\
2020\end{array}$ & NR & $56 / 56$ & 61.11 & 61.15 & CT, DHI & CT & $\begin{array}{l}\text { Once } \\
\text { daily, } \\
30 \mathrm{ml}\end{array}$ & $14 d$ & (1) (14) \\
\hline $\begin{array}{l}\text { Liu } \\
2018\end{array}$ & NR & $83 / 83$ & NR & NR & Edaravone, DHI & Edaravone & $\begin{array}{l}\text { Once } \\
\text { daily, 20- } \\
40 \mathrm{ml}\end{array}$ & $14 d$ & (1) (14) \\
\hline $\begin{array}{l}\text { Shi } \\
2018\end{array}$ & $\begin{array}{l}\text { Acute } \\
\text { phase }\end{array}$ & $40 / 40$ & 64.00 & 65.00 & Alprostadil, DHI & Alprostadil & $\begin{array}{l}\text { Once } \\
\text { daily, } \\
40 \mathrm{ml}\end{array}$ & $14 d$ & $(1)(4)$ \\
\hline $\begin{array}{l}\text { Yang } \\
2018\end{array}$ & $\begin{array}{l}\text { Acute } \\
\text { phase }\end{array}$ & $47 / 47$ & NR & NR & Edaravone, DHI & Edaravone & $\begin{array}{l}\text { Once } \\
\text { daily, } \\
40 \mathrm{ml}\end{array}$ & $14 d$ & $\left(\begin{array}{l}1)(3)(8) \\
9\end{array}\right.$ \\
\hline $\begin{array}{l}\text { Ma } \\
2017\end{array}$ & NR & $40 / 42$ & 60.01 & 65.26 & $\mathrm{CT}, \mathrm{DHI}$ & CT & $\begin{array}{l}\text { Once } \\
\text { daily, } \\
20 \mathrm{ml}\end{array}$ & $14 d$ & $\begin{array}{l}(1)(2) \\
(13)\end{array}$ \\
\hline
\end{tabular}

$\mathrm{CG}=$ control group; $\mathrm{EG}=$ experiment group (Danhong Injection group); NR=not reported; $\mathrm{CT}=$ conventional treatment.

Outcomes: (1)=NIHSS, (2)=Fugl-Meyer Assessment, (3)=Barthel index, (4)=ADL, (5)=IMT, (6)=cerebral blood flow, (7)=average cerebral blood flow rate, (8)=total cholesterol, (9)=triglycerides, (10)=HDL, (11)=LDL, (12)=Hcy, (13)=D-dimer, (14)=ADRs. 


\begin{tabular}{|c|c|c|c|c|c|c|c|c|c|}
\hline \multirow[t]{2}{*}{$\begin{array}{l}\text { Author } \\
\text { (year) }\end{array}$} & \multirow{2}{*}{$\begin{array}{l}\text { Phase } \\
\text { of } \\
\text { disease }\end{array}$} & \multirow{2}{*}{$\begin{array}{l}\text { Sample } \\
\text { size, } \\
\text { EG/CG }\end{array}$} & \multicolumn{2}{|c|}{$\begin{array}{l}\text { Mean age } \\
\text { (year) }\end{array}$} & \multicolumn{2}{|l|}{ Interventions } & \multirow{2}{*}{$\begin{array}{l}\text { Dosages } \\
\text { of } \\
\text { Danhong } \\
\text { Injection }\end{array}$} & \multirow[t]{2}{*}{ Course } & \multirow[t]{2}{*}{ Outcomes } \\
\hline & & & EG & CG & EG & CG & & & \\
\hline $\begin{array}{l}\text { Liu } \\
2010\end{array}$ & $\begin{array}{l}\text { Acute } \\
\text { phase }\end{array}$ & $44 / 43$ & 63.70 & 64.40 & $\begin{array}{l}\text { Fasudil } \\
\text { Hydrochloride, DHI }\end{array}$ & $\begin{array}{l}\text { Fasudil } \\
\text { Hydrochloride }\end{array}$ & $\begin{array}{l}\text { Once } \\
\text { daily, } \\
30 \mathrm{ml}\end{array}$ & $14 d$ & (4) (14) \\
\hline $\begin{array}{l}\text { Chen } \\
2017\end{array}$ & NR & $32 / 32$ & 53.12 & 52.93 & $\mathrm{CT}, \mathrm{DHI}$ & CT & $\begin{array}{l}\text { Once } \\
\text { daily, } \\
30 \mathrm{ml}\end{array}$ & $7 d$ & (8) \\
\hline $\begin{array}{l}\text { Zhang } \\
2018\end{array}$ & $\begin{array}{l}\text { Acute } \\
\text { phase }\end{array}$ & $54 / 54$ & 61.80 & 60.20 & Alprostadil, DHI & Alprostadil & $\begin{array}{l}\text { Once } \\
\text { daily, } \\
30 \mathrm{ml}\end{array}$ & $14 d$ & (1) (14) \\
\hline $\begin{array}{l}\text { Zhang } \\
2017\end{array}$ & $\begin{array}{l}\text { Acute } \\
\text { phase }\end{array}$ & $45 / 45$ & 68.90 & 69.30 & Edaravone, DHI & Edaravone & $\begin{array}{l}\text { Twice } \\
\text { daily, } \\
20 \mathrm{ml}\end{array}$ & $14 d$ & (14) \\
\hline $\begin{array}{l}\text { Pen } \\
2017\end{array}$ & NR & $39 / 39$ & NR & NR & $\begin{array}{l}\text { Atorvastatin } \\
\text { Calcium, DHI }\end{array}$ & $\begin{array}{l}\text { Atorvastatin } \\
\text { Calcium }\end{array}$ & $\begin{array}{l}\text { Once } \\
\text { daily, } \\
10 \mathrm{ml}\end{array}$ & $14 d$ & (5) \\
\hline $\begin{array}{l}\text { Fan } \\
2018\end{array}$ & $\begin{array}{l}\text { Acute } \\
\text { phase }\end{array}$ & $35 / 35$ & 49.32 & 50.45 & $\mathrm{CT}, \mathrm{DHI}$ & CT & $\begin{array}{l}\text { Once } \\
\text { daily, } \\
40 \mathrm{ml}\end{array}$ & $14 d$ & (1) (4) \\
\hline Li 2017 & $\begin{array}{l}\text { Acute } \\
\text { phase }\end{array}$ & $48 / 48$ & 55.76 & 56.37 & Clopidogrel, DHI & Clopidogrel & $\begin{array}{l}\text { Once } \\
\text { daily, } \\
30 \mathrm{ml}\end{array}$ & $14 d$ & $\begin{array}{l}(1)(4) \\
(14)\end{array}$ \\
\hline $\begin{array}{l}\text { Jiang } \\
2020\end{array}$ & $\begin{array}{l}\text { Acute } \\
\text { phase }\end{array}$ & $60 / 60$ & 60.47 & 59.82 & $\begin{array}{l}\text { rt-PA, Sodium } \\
\text { Heparin, DHI }\end{array}$ & $\begin{array}{l}\text { rt-PA, Sodium } \\
\text { Heparin }\end{array}$ & $\begin{array}{l}\text { Once } \\
\text { daily, } \\
20 \mathrm{ml}\end{array}$ & $14 d$ & $\begin{array}{l}(1)(5) \\
14)^{(5)}\end{array}$ \\
\hline $\begin{array}{l}\text { Liu } \\
2019\end{array}$ & $\begin{array}{l}\text { Acute } \\
\text { phase }\end{array}$ & $56 / 56$ & 56.91 & 55.73 & Edaravone, DHI & Edaravone & $\begin{array}{l}\text { Once } \\
\text { daily, } \\
20 \mathrm{mg}\end{array}$ & $14 d$ & $(2)(3)(6)$ \\
\hline $\begin{array}{l}\text { Liu } \\
2017\end{array}$ & NR & $30 / 30$ & 60.24 & 58.49 & $\mathrm{CT}, \mathrm{DHI}$ & CT & $\begin{array}{l}\text { Once } \\
\text { daily, } \\
30 \mathrm{ml}\end{array}$ & $14 d$ & (13) \\
\hline $\begin{array}{l}\mathrm{Ge} \\
2018\end{array}$ & NR & $37 / 37$ & 62.13 & 61.52 & $\begin{array}{l}\text { Atorvastatin } \\
\text { Calcium, DHI }\end{array}$ & $\begin{array}{l}\text { Atorvastatin } \\
\text { Calcium }\end{array}$ & $\begin{array}{l}\text { Once } \\
\text { daily, } \\
20 \mathrm{ml}\end{array}$ & $14 d$ & (1) (14) \\
\hline $\begin{array}{l}\text { Yun } \\
2017\end{array}$ & $\begin{array}{l}\text { Acute } \\
\text { phase }\end{array}$ & $31 / 31$ & 69.50 & 68.20 & Aspirin, DHI & Aspirin & $\begin{array}{l}\text { Once } \\
\text { daily, } \\
30 \mathrm{ml}\end{array}$ & $14 d$ & (7) \\
\hline $\begin{array}{l}\text { Liu } \\
2019\end{array}$ & $\begin{array}{l}\text { Acute } \\
\text { phase }\end{array}$ & $33 / 32$ & 41.84 & 42.53 & $\begin{array}{l}\text { Butylphthalide soft } \\
\text { capsules, DHI }\end{array}$ & $\begin{array}{l}\text { Butylphthalide } \\
\text { soft capsules }\end{array}$ & $\begin{array}{l}\text { Once } \\
\text { daily, } \\
40 \mathrm{ml}\end{array}$ & $14 d$ & (1) \\
\hline $\begin{array}{l}\text { Dai } \\
2018\end{array}$ & $\begin{array}{l}\text { Acute } \\
\text { phase }\end{array}$ & $47 / 47$ & 56.42 & 56.43 & $\begin{array}{l}\text { Aspirin,Nimodipine, } \\
\text { DHI }\end{array}$ & Aspirin,Nimodipine & $\begin{array}{l}\text { Twice } \\
\text { daily, } \\
40 \mathrm{ml}\end{array}$ & $15 d$ & $\begin{array}{l}(1)(4)(8) \\
(9)\end{array}$ \\
\hline $\begin{array}{l}\text { Yang } \\
2018\end{array}$ & $\begin{array}{l}\text { Acute } \\
\text { phase }\end{array}$ & $39 / 39$ & 64.08 & 63.22 & $\begin{array}{l}\text { Atorvastatin } \\
\text { Calcium, DHI }\end{array}$ & $\begin{array}{l}\text { Atorvastatin } \\
\text { Calcium }\end{array}$ & $\begin{array}{l}\text { Once } \\
\text { daily, } \\
20 \mathrm{ml}\end{array}$ & $14 d$ & (1) (3) \\
\hline Li 2015 & $\begin{array}{l}\text { Acute } \\
\text { phase }\end{array}$ & $105 / 105$ & 71.30 & 71.60 & Edaravone, DHI & Edaravone & $\begin{array}{l}\text { Once } \\
\text { daily, } \\
30 \mathrm{ml}\end{array}$ & $14 d$ & (1) (8) (9) \\
\hline
\end{tabular}

$\mathrm{CG}=$ control group; $\mathrm{EG}=$ experiment group (Danhong Injection group); $\mathrm{NR}=$ not reported; $\mathrm{CT}=$ conventional treatment.

Outcomes: (1)=NIHSS, (2)=Fugl-Meyer Assessment, (3)=Barthel index, (4)=ADL, (5)=IMT, (6)=cerebral blood flow, (7)=average cerebral blood flow rate, (8)=total cholesterol, (9)=triglycerides, (10)=HDL, (11)=LDL, (12)=Hcy, (13)=D-dimer, (14)=ADRs. 


\begin{tabular}{|c|c|c|c|c|c|c|c|c|c|}
\hline \multirow[t]{2}{*}{$\begin{array}{l}\text { Author } \\
\text { (year) }\end{array}$} & \multirow{2}{*}{$\begin{array}{l}\text { Phase } \\
\text { of } \\
\text { disease }\end{array}$} & \multirow{2}{*}{$\begin{array}{l}\text { Sample } \\
\text { size, } \\
\text { EG/CG }\end{array}$} & \multicolumn{2}{|c|}{$\begin{array}{l}\text { Mean age } \\
\text { (year) }\end{array}$} & \multicolumn{2}{|l|}{ Interventions } & \multirow{2}{*}{$\begin{array}{l}\text { Dosages } \\
\text { of } \\
\text { Danhong } \\
\text { Injection }\end{array}$} & \multirow[t]{2}{*}{ Course } & \multirow[t]{2}{*}{ Outcomes } \\
\hline & & & EG & CG & EG & CG & & & \\
\hline Li 2020 & $\begin{array}{l}\text { Acute } \\
\text { phase }\end{array}$ & $52 / 52$ & 68.34 & 68.58 & $\begin{array}{l}\text { 3-n-butylphthalide, } \\
\text { DHI }\end{array}$ & 3-n-butylphthalide & $\begin{array}{l}\text { Once } \\
\text { daily, } \\
30 \mathrm{ml}\end{array}$ & $14 d$ & (1) (7) \\
\hline $\begin{array}{l}\text { Liu } \\
2017\end{array}$ & $\begin{array}{l}\text { Acute } \\
\text { phase }\end{array}$ & $54 / 55$ & 56.83 & 56.94 & $\mathrm{CT}, \mathrm{DHI}$ & CT & $\begin{array}{l}\text { Once } \\
\text { daily, } \\
20 \mathrm{ml}\end{array}$ & $14 d$ & (1) \\
\hline $\begin{array}{l}\text { Luo } \\
2018\end{array}$ & $\begin{array}{l}\text { Acute } \\
\text { phase }\end{array}$ & $56 / 56$ & 65.40 & 65.10 & $\mathrm{CT}, \mathrm{DHI}$ & $\mathrm{CT}$ & $\begin{array}{l}\text { Twice } \\
\text { daily, } \\
\text { 30mi }\end{array}$ & $28 d$ & (2) (14) \\
\hline $\begin{array}{l}\text { Kang } \\
2020\end{array}$ & $\begin{array}{l}\text { Acute } \\
\text { phase }\end{array}$ & $65 / 60$ & 55.42 & 54.95 & $\begin{array}{l}\text { Tirofiban } \\
\text { Hydrochloride, } \\
\text { Clopidogrel, } \\
\text { Aspirin, DHI }\end{array}$ & $\begin{array}{l}\text { Tirofiban } \\
\text { Hydrochloride, } \\
\text { Clopidogrel, } \\
\text { Aspirin }\end{array}$ & $\begin{array}{l}\text { Once } \\
\text { daily, } \\
20 \mathrm{ml}\end{array}$ & $14 d$ & $\begin{array}{l}(1)(4) \\
(14)\end{array}$ \\
\hline $\begin{array}{l}\text { Yang } \\
2018\end{array}$ & $\begin{array}{l}\text { Acute } \\
\text { phase }\end{array}$ & $58 / 58$ & 57.86 & 57.09 & Clopidogrel, DHI & Clopidogrel & $\begin{array}{l}\text { Once } \\
\text { daily, } \\
30 \mathrm{ml}\end{array}$ & $14 d$ & (1) (14) \\
\hline $\begin{array}{l}\text { Liu } \\
2020\end{array}$ & $\begin{array}{l}\text { Acute } \\
\text { phase }\end{array}$ & $71 / 71$ & 63.24 & 61.97 & Edaravone, DHI & Edaravone & $\begin{array}{l}\text { Once } \\
\text { daily, } \\
20 \mathrm{ml}\end{array}$ & $14 d$ & (1) (3) (7) \\
\hline $\begin{array}{l}\text { Wang } \\
2016\end{array}$ & $\begin{array}{l}\text { Acute } \\
\text { phase }\end{array}$ & $56 / 56$ & NR & NR & $\mathrm{CT}, \mathrm{DHI}$ & CT & $\begin{array}{l}\text { Once } \\
\text { daily, } \\
450 \mathrm{mg}\end{array}$ & $168 d$ & (1) \\
\hline $\begin{array}{l}\text { Yuan } \\
2019\end{array}$ & $\begin{array}{l}\text { Acute } \\
\text { phase }\end{array}$ & $38 / 38$ & 65.50 & 65.39 & Edaravone, DHI & Edaravone & $\begin{array}{l}\text { Once } \\
\text { daily, } \\
40 \mathrm{ml}\end{array}$ & $14 d$ & $\begin{array}{l}(1)(2)(3) \\
(12)\end{array}$ \\
\hline $\begin{array}{l}\text { Su } \\
2012\end{array}$ & $\begin{array}{l}\text { Acute } \\
\text { phase }\end{array}$ & $38 / 37$ & 61.58 & 63.01 & $\mathrm{CT}, \mathrm{DHI}$ & $\mathrm{CT}$ & $\begin{array}{l}\text { Once } \\
\text { daily, } \\
40 \mathrm{ml}\end{array}$ & $14 d$ & $\begin{array}{l}(8)(9) \\
(11)(14)\end{array}$ \\
\hline $\begin{array}{l}\text { Luo } \\
2012\end{array}$ & $\begin{array}{l}\text { Acute } \\
\text { phase }\end{array}$ & $90 / 90$ & NR & NR & $\begin{array}{l}\text { Ozagrel Sodium, } \\
\text { DHI }\end{array}$ & Ozagrel Sodium & $\begin{array}{l}\text { Once } \\
\text { daily, } \\
40 \mathrm{ml}\end{array}$ & $14 d$ & (1) \\
\hline $\begin{array}{l}\text { Zou } \\
2013\end{array}$ & $\begin{array}{l}\text { Acute } \\
\text { phase }\end{array}$ & $40 / 40$ & 58.70 & 57.90 & Vinpocetine, DHI & Vinpocetine & $\begin{array}{l}\text { Once } \\
\text { daily, } \\
30 \mathrm{ml}\end{array}$ & $14 d$ & (1) \\
\hline $\begin{array}{l}\text { Liang } \\
2014\end{array}$ & $\begin{array}{l}\text { Acute } \\
\text { phase }\end{array}$ & $45 / 45$ & 53.50 & 52.60 & $\mathrm{CT}, \mathrm{DHI}$ & CT & $\begin{array}{l}\text { Once } \\
\text { daily, } \\
30 \mathrm{ml}\end{array}$ & $14 d$ & (4) \\
\hline $\begin{array}{l}\mathrm{Ma} \\
2015\end{array}$ & $\begin{array}{l}\text { Acute } \\
\text { phase }\end{array}$ & $45 / 45$ & 65.37 & 65.64 & $\mathrm{CT}, \mathrm{DHI}$ & CT & $\begin{array}{l}\text { Once } \\
\text { daily, } \\
20 \mathrm{ml}\end{array}$ & $28 d$ & (2) (3) \\
\hline $\begin{array}{l}\text { Fan } \\
2015\end{array}$ & NR & $45 / 45$ & 62.30 & 61.50 & $\mathrm{CT}, \mathrm{DHI}$ & $\mathrm{CT}$ & $\begin{array}{l}\text { Once } \\
\text { daily, } \\
20 \mathrm{ml}\end{array}$ & $28 d$ & $\begin{array}{l}(8)(9) \\
(10)(11)\end{array}$ \\
\hline $\begin{array}{l}\text { Zeng } \\
2016\end{array}$ & $\begin{array}{l}\text { Acute } \\
\text { phase }\end{array}$ & $55 / 55$ & 63.00 & 63.30 & Edaravone, $\mathrm{DHI}$ & Edaravone & $\begin{array}{l}\text { Once } \\
\text { daily, } \\
20 \mathrm{ml}\end{array}$ & $15 d$ & (3) \\
\hline $\begin{array}{l}\text { Feng } \\
2016\end{array}$ & $\begin{array}{l}\text { Acute } \\
\text { phase }\end{array}$ & $20 / 20$ & 59.82 & 60.50 & rt-PA, DHI & rt-PA & $\begin{array}{l}\text { Once } \\
\text { daily, } \\
20 \mathrm{ml}\end{array}$ & $14 d$ & (3) (13) \\
\hline \multicolumn{10}{|c|}{$\mathrm{CG}=$ control group; $\mathrm{EG}=$ experiment group (Danhong Injection group); $\mathrm{NR}=$ not reported; $\mathrm{CT}=$ conventional treatment. } \\
\hline $\begin{array}{l}\text { utcon } \\
\text { rebra }\end{array}$ & $=\mathrm{NIH}$ & 2) $=\mathrm{Fu}$ & er $A$ & sme & 3) $=$ Barthel index & $\mathrm{DL},(5)=\mathrm{IMT},(6)$ & $\begin{array}{l}\text { ral bloo } \\
=\text { D-dim }\end{array}$ & $v_{,}(7)$ & age \\
\hline
\end{tabular}




\begin{tabular}{|c|c|c|c|c|c|c|c|c|c|}
\hline \multirow[t]{2}{*}{$\begin{array}{l}\text { Author } \\
\text { (year) }\end{array}$} & \multirow{2}{*}{$\begin{array}{l}\text { Phase } \\
\text { of } \\
\text { disease }\end{array}$} & \multirow{2}{*}{$\begin{array}{l}\text { Sample } \\
\text { size, } \\
\text { EG/CG }\end{array}$} & \multicolumn{2}{|c|}{$\begin{array}{l}\text { Mean age } \\
\text { (year) }\end{array}$} & \multicolumn{2}{|l|}{ Interventions } & \multirow{2}{*}{$\begin{array}{l}\text { Dosages } \\
\text { of } \\
\text { Danhong } \\
\text { Injection }\end{array}$} & \multirow[t]{2}{*}{ Course } & \multirow[t]{2}{*}{ Outcomes } \\
\hline & & & EG & CG & EG & CG & & & \\
\hline $\begin{array}{l}\mathrm{Ou} \\
2017\end{array}$ & $\begin{array}{l}\text { Acute } \\
\text { phase }\end{array}$ & $60 / 60$ & 58.10 & 57.20 & $\mathrm{CT}, \mathrm{DHI}$ & $\mathrm{CT}$ & $\begin{array}{l}\text { Once } \\
\text { daily, } \\
20 \mathrm{ml}\end{array}$ & $14 d$ & $\begin{array}{l}(1)(4) \\
(12)\end{array}$ \\
\hline $\begin{array}{l}\text { Wu } \\
2016\end{array}$ & $\begin{array}{l}\text { Acute } \\
\text { phase }\end{array}$ & $49 / 49$ & 67.40 & 68.10 & $\begin{array}{l}\text { Alprostadil, } \\
\text { Edaravone, } \\
\text { Aspirin, DHI }\end{array}$ & $\begin{array}{l}\text { Alprostadil, } \\
\text { Edaravone, } \\
\text { Aspirin }\end{array}$ & $\begin{array}{l}\text { Once } \\
\text { daily, } \\
40 \mathrm{ml}\end{array}$ & $14 d$ & $\begin{array}{l}(1)(3) \\
(12)(14)\end{array}$ \\
\hline $\begin{array}{l}\text { Chen } \\
2017\end{array}$ & $\begin{array}{l}\text { Acute } \\
\text { phase }\end{array}$ & $40 / 40$ & 67.34 & 66.46 & $\mathrm{CT}, \mathrm{DHI}$ & CT & $\begin{array}{l}\text { Once } \\
\text { daily, } \\
20 \mathrm{ml}\end{array}$ & $14 d$ & (2) (14) \\
\hline $\begin{array}{l}\text { Qiu } \\
2017\end{array}$ & $\begin{array}{l}\text { Acute } \\
\text { phase }\end{array}$ & $58 / 58$ & 70.69 & 69.88 & $\mathrm{CT}, \mathrm{DHI}$ & $\mathrm{CT}$ & $\begin{array}{l}\text { Once } \\
\text { daily, } \\
30 \mathrm{ml}\end{array}$ & $14 d$ & $\begin{array}{l}(1)(4)(8) \\
(9)\end{array}$ \\
\hline $\begin{array}{l}\text { Liu } \\
2017\end{array}$ & $\begin{array}{l}\text { Acute } \\
\text { phase }\end{array}$ & $48 / 48$ & 52.33 & 52.05 & $\mathrm{CT}, \mathrm{DHI}$ & $\mathrm{CT}$ & $\begin{array}{l}\text { Once } \\
\text { daily, } \\
20 \mathrm{ml}\end{array}$ & $14 d$ & (1) \\
\hline $\begin{array}{l}\text { Wei } \\
2017\end{array}$ & $\begin{array}{l}\text { Acute } \\
\text { phase }\end{array}$ & $47 / 47$ & 62.60 & 60.73 & $\mathrm{CT}, \mathrm{DHI}$ & CT & $\begin{array}{l}\text { Once } \\
\text { daily, } \\
20 \mathrm{ml}\end{array}$ & $14 d$ & (1) (14) \\
\hline $\begin{array}{l}\text { Jing } \\
2020\end{array}$ & $\begin{array}{l}\text { Acute } \\
\text { phase }\end{array}$ & $44 / 44$ & 62.70 & 63.20 & $\mathrm{CT}, \mathrm{DHI}$ & CT & $\begin{array}{l}\text { Once } \\
\text { daily, } \\
20 \mathrm{ml}\end{array}$ & $14 d$ & $\begin{array}{l}(1)(3)(6) \\
(7)\end{array}$ \\
\hline Li 2018 & $\begin{array}{l}\text { Acute } \\
\text { phase }\end{array}$ & $50 / 50$ & 51.90 & 52.30 & $\begin{array}{l}\text { Atorvastatin } \\
\text { Calcium, DHI }\end{array}$ & $\begin{array}{l}\text { Atorvastatin } \\
\text { Calcium }\end{array}$ & $\begin{array}{l}\text { Once } \\
\text { daily, } \\
20 \mathrm{ml}\end{array}$ & $14 d$ & (14) \\
\hline $\begin{array}{l}\text { Jin } \\
2019\end{array}$ & $\begin{array}{l}\text { Acute } \\
\text { phase }\end{array}$ & $50 / 50$ & 52.00 & 54.00 & rt-PA, DHI & $\mathrm{rt}-\mathrm{PA}$ & $\begin{array}{l}\text { Once } \\
\text { daily, } \\
20 \mathrm{ml}\end{array}$ & $14 d$ & (1) \\
\hline $\begin{array}{l}\text { Chai } \\
2019\end{array}$ & $\begin{array}{l}\text { Acute } \\
\text { phase }\end{array}$ & $42 / 42$ & 64.08 & 63.17 & $\begin{array}{l}\text { Ozagrel Sodium, } \\
\text { Aspirin, DHI }\end{array}$ & $\begin{array}{l}\text { Ozagrel Sodium, } \\
\text { Aspirin }\end{array}$ & $\begin{array}{l}\text { Once } \\
\text { daily, } \\
40 \mathrm{ml}\end{array}$ & $14 d$ & (1) (4) \\
\hline $\begin{array}{l}\text { Chen } \\
2019\end{array}$ & $\begin{array}{l}\text { Acute } \\
\text { phase }\end{array}$ & $74 / 74$ & 54.69 & 52.61 & $\mathrm{CT}, \mathrm{DHI}$ & CT & $\begin{array}{l}\text { Once } \\
\text { daily, } \\
20 \mathrm{ml}\end{array}$ & $14 d$ & (1) \\
\hline Li 2019 & $\begin{array}{l}\text { Acute } \\
\text { phase }\end{array}$ & $42 / 42$ & 63.49 & 63.58 & $\begin{array}{l}\text { Urinary } \\
\text { Kallidinogenase, } \\
\text { DHI }\end{array}$ & $\begin{array}{l}\text { Urinary } \\
\text { Kallidinogenase }\end{array}$ & $\begin{array}{l}\text { Once } \\
\text { daily, } \\
20 \mathrm{ml}\end{array}$ & $14 d$ & (1) (14) \\
\hline $\begin{array}{l}\text { Zhu } \\
2020\end{array}$ & $\begin{array}{l}\text { Acute } \\
\text { phase }\end{array}$ & $30 / 30$ & 56.34 & 57.62 & $\mathrm{CT}, \mathrm{DHI}$ & $\mathrm{CT}$ & $\begin{array}{l}\text { Once } \\
\text { daily, } \\
20 \mathrm{ml}\end{array}$ & $14 d$ & (1) (3) \\
\hline
\end{tabular}

$\mathrm{CG}=$ control group; $\mathrm{EG}=$ experiment group (Danhong Injection group); $\mathrm{NR}=$ not reported; $\mathrm{CT}=$ conventional treatment.

Outcomes: (1)=NIHSS, (2)=Fugl-Meyer Assessment, (3)=Barthel index, (4)=ADL, (5)=IMT, (6)=cerebral blood flow, (7)=average cerebral blood flow rate, (8)=total cholesterol, (9)=triglycerides, (10)=HDL, (11)=LDL, (12)=Hcy, (13)=D-dimer, (14)=ADRs. 


\begin{tabular}{|c|c|c|c|c|c|c|c|c|c|}
\hline \multirow[t]{2}{*}{$\begin{array}{l}\text { Author } \\
\text { (year) }\end{array}$} & \multirow{2}{*}{$\begin{array}{l}\text { Phase } \\
\text { of } \\
\text { disease }\end{array}$} & \multirow{2}{*}{$\begin{array}{l}\text { Sample } \\
\text { size, } \\
\text { EG/CG }\end{array}$} & \multicolumn{2}{|c|}{$\begin{array}{l}\text { Mean age } \\
\text { (year) }\end{array}$} & \multicolumn{2}{|l|}{ Interventions } & \multirow{2}{*}{$\begin{array}{l}\text { Dosages } \\
\text { of } \\
\text { Danhong } \\
\text { Injection }\end{array}$} & \multirow[t]{2}{*}{ Course } & \multirow[t]{2}{*}{ Outcomes } \\
\hline & & & EG & CG & EG & CG & & & \\
\hline $\begin{array}{l}\text { Cao } \\
2019\end{array}$ & $\begin{array}{l}\text { Acute } \\
\text { phase }\end{array}$ & $150 / 150$ & 59.70 & 59.00 & CT, DHI & CT & $\begin{array}{l}\text { Once } \\
\text { daily, } \\
\text { 30ml }\end{array}$ & $14 d$ & (13) (14) \\
\hline $\begin{array}{l}\text { Song } \\
2014\end{array}$ & $\begin{array}{l}\text { Acute } \\
\text { phase }\end{array}$ & $65 / 65$ & 65.23 & 65.89 & CT, DHI & CT & $\begin{array}{l}\text { Once } \\
\text { daily, } \\
30 \mathrm{ml}\end{array}$ & $14 d$ & $\begin{array}{l}(1)(3)(8) \\
(9)(11) \\
(13)(14)\end{array}$ \\
\hline $\begin{array}{l}\text { Lv } \\
2018\end{array}$ & $\begin{array}{l}\text { Acute } \\
\text { phase }\end{array}$ & $40 / 40$ & 63.01 & 62.34 & rt-PA & $\mathrm{rt}-\mathrm{PA}$ & $\begin{array}{l}\text { Once } \\
\text { daily, } \\
20 \mathrm{ml}\end{array}$ & $14 d$ & (3) (14) \\
\hline $\begin{array}{l}\text { Huo } \\
2020\end{array}$ & $\begin{array}{l}\text { Acute } \\
\text { phase }\end{array}$ & $45 / 45$ & NR & NR & $\begin{array}{l}\text { Deproteinised Calf } \\
\text { Blood Injection, } \\
\text { DHI }\end{array}$ & $\begin{array}{l}\text { Deproteinised Calf } \\
\text { Blood Injection }\end{array}$ & $\begin{array}{l}\text { Once } \\
\text { daily, } \\
30 \mathrm{mg}\end{array}$ & $14 d$ & (1) \\
\hline $\begin{array}{l}\text { Cao } \\
2016\end{array}$ & $\begin{array}{l}\text { Acute } \\
\text { phase }\end{array}$ & $34 / 33$ & 54.50 & 58.90 & CT, DHI & CT & $\begin{array}{l}\text { Once } \\
\text { daily, } \\
30 \mathrm{ml}\end{array}$ & $14 d$ & (1) (14) \\
\hline $\begin{array}{l}\text { Ren } \\
2011\end{array}$ & $\begin{array}{l}\text { Acute } \\
\text { phase }\end{array}$ & $34 / 34$ & 62.20 & 63.10 & CT, DHI & CT & $\begin{array}{l}\text { Once } \\
\text { daily, } \\
40 \mathrm{ml}\end{array}$ & $14 d$ & (1) \\
\hline $\begin{array}{l}\text { Xue } \\
2010\end{array}$ & NR & $37 / 49$ & 65.70 & 67.50 & CT, DHI & CT & $\begin{array}{l}\text { Once } \\
\text { daily, } \\
30 \mathrm{ml}\end{array}$ & $14 d$ & $\begin{array}{l}(5)(8) \\
(10)(11) \\
(14)\end{array}$ \\
\hline \multicolumn{10}{|c|}{ 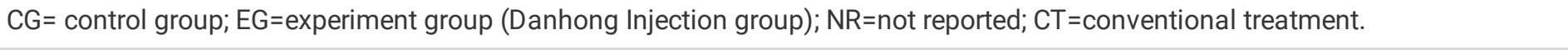 } \\
\hline $\begin{array}{l}\text { Outcol } \\
\text { cerebr }\end{array}$ & $\begin{array}{l}\text { ) }=\mathrm{NIH} \\
\mathrm{d} \text { flon }\end{array}$ & 2) $=$ Fug & er & sm & $\begin{array}{l}\text { 3)=Barthel index } \\
\text { lycerides, }(10)=1\end{array}$ & $\mathrm{~L},(5)=\mathrm{IMT},(6)$ & al blo & $(7$ & age \\
\hline
\end{tabular}

All RCTs reported methods of random sequences generation. None of the trials mentioned allocation concealment. Only three trials [31, 39,52 ] (4\%) blinded patients and caregivers. No trials mentioned loss to follow-up. No details were identified in the domains of other bias (Supplementary table 1).

\section{Treatment effects}

In total, 45 RCTs $[8,16,19-21,23-30,33,36-38,40,42-46,48-51,53,54,60,61,63-66,68-72,74,76-78]$ involving 4565 patients reported NIHSS score. For Danhong injection combined with western medicine group, the NIHSS score significantly changed -10.50 (95\% Cl: -11.50囚-9.51), and the control group changed -6.22 (95\% Cl: -7.05囚-5.39). Meta-analysis showed that Dahong injection combined with western medicine had a better effect in improving neurological impairment than western medicine alone (NIHSS score MD $-4.21,95 \% \mathrm{Cl}-4.96 \rrbracket-3.46$ )(Figure 2).

Six RCTs[6, 30, 47, 51, 56, 62] involving 552 patients showed that Danhong injection combined with western medicine could significantly improve the Fugl-Meyer Assessment than western medicine alone (MD 14.28, 95\% Cl 9.47ه19.09) (Figure 3). Compared with western medicine alone, Danhong injection plus western medicine suggested a significant improvement on Barthel index (14 trials with 1270 patients; MD 8.71, 95\% Cl 3.68ه13.74) and ADL score (12 trials with 1114 patients; MD 14.48, 95\% Cl 9.04ه19.92) (Figure S1 and $\mathrm{S} 2$ in appendix 3).

Meta-analysis showed that compared with western medicine alone, combination therapy of Danhong injection and western medicine could significantly lower the IMT ( 3 trials with 284 patients; MD $-0.23 \mathrm{~mm}, 95 \% \mathrm{Cl}-0.26 \mathrm{X}-0.20$ ), increase the cerebral blood flow ( 2 trials with 200 patients; MD $1.16 \mathrm{~mL} / \mathrm{s}, 95 \% \mathrm{Cl} 0.64 \otimes 1.68$ ) and average cerebral blood flow rate ( 5 trials with 508 patients; MD $3.05 \mathrm{~cm} / \mathrm{s}$, 95\% Cl 1.61ه4.50) (Figures S3-S5 in appendix 3). 
The combination therapy of Danhong injection and western medicine could significantly lower total cholesterol (10 trials with 1019 patients; MD -1.14 mmol/L, 95\% Cl -1.57ه-0.72) (Figure 4), triglycerides (8 trials with 869 patients; MD $-1.00 \mathrm{mmol} / \mathrm{L}, 95 \% \mathrm{Cl}-1.69$ $\bigotimes-0.31$ ) and LDL (5 trials with 441 patients; $M D-0.91 \mathrm{mmol} / \mathrm{L}, 95 \% \mathrm{Cl}-1.33 \rrbracket-0.49$ ), and increase the level of HDL (3 trials with 236 patients; MD 0.31 mmol/L, 95\% Cl 0.22ه0.40) (Figures S6-S8 in appendix 3).

Four RCTs[16, 51, 60, 61] with a total of 392 individuals reported the data of serum Hcy level. The results revealed a significant

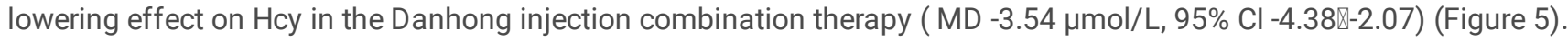

Meta-analysis of five trials with a total of 612 patients showed no statistically significant difference in D-dimer (MD $-0.12 \mathrm{mg} / \mathrm{L}, 95 \% \mathrm{Cl}$ $-0.61 \otimes 0.37, \mathrm{P}=0.64$ ) between treatment groups (Figure $\mathrm{S} 9$ in appendix 3 ).

\section{Subgroup Analysis}

Subgroup analysis by mean age showed differential effects in average cerebral blood flow rate ( $<60$ years old: $\mathrm{MD} 0.74 \mathrm{~cm} / \mathrm{s}, 95 \% \mathrm{Cl}$ $0.29 \otimes 1.19 ; \geq 60$ years old: $\mathrm{MD} 4.09 \mathrm{~cm} / \mathrm{s}, 95 \% \mathrm{Cl} 2.02 \varangle 6.16$; interaction $\mathrm{P}=0.002$ ). The analysis by length of treatment showed differential effects in ADL ( $\leq 2$ weeks: MD 15.08, 95\% Cl 9.11ष21.05; >2 weeks: MD 8.18, 95\% Cl 6.21ه10.15; interaction P=0.03). Subgroup analysis by type of baseline western medicines showed differential effects (conventional therapy: $\mathrm{MD}-3.79,95 \% \mathrm{Cl}-4.83$

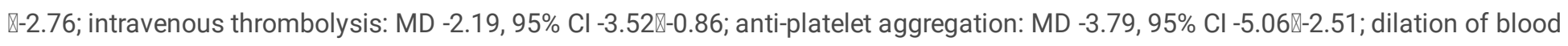
vessels: $\mathrm{MD}-4.23,95 \% \mathrm{Cl}-5.85 \rrbracket-2.62$; statins: $\mathrm{MD}-2.87,95 \% \mathrm{Cl}-4.07 \rrbracket-1.67$; neuroprotective agents : $\mathrm{MD}-5.88,95 \% \mathrm{Cl}-8.90 \rrbracket-2.87$; other therapies : $\mathrm{MD}-10.20,95 \% \mathrm{Cl}-12.54 \rrbracket-7.86$; interaction $\mathrm{P}<0.00001)$.

As for Fugl-Meyer Assessment, Barthel index, total cholesterol, triglycerides, LDL, Hcy and D-dimer, subgroup analyses by length of treatment, mean age of patients, and types of baseline western medicine treatment showed no statistically differential effects.

\section{Safety}

Of the 67 included trials, 16 studies [20, 22, 25-27, 31, 33, 34, 37, 40, 49, 62, 65, 67, 71, 75] reported at least one case of ADRs, 12 studies $[16-18,38,47,48,52,61,73,74,77,79]$ reported no ADRs/ADEs during the study, and 39 RCTs did not mention the information of ADRs/ADEs.

The main ADRs occurred during the trials were gastrointestinal reactions( $4.48 \%$ vs $4.76 \%$ in Danhong injection plus western medicine and western medicine alone group, respectively, $\mathrm{P}=0.76)$, dizzy ( $3.69 \%$ vs $2.74 \%$ in each group, $\mathrm{P}=0.61)$ and skin rash $(8.53 \%$ vs $7.31 \%$ in each group, $\mathrm{P}=0.56$ ). ADRs occurred during the trials in either group were shown in Supplementary table 2.

\section{Assessment of Publication Bias}

The funnel plots for NIHSS, Barthel index and ADL were near asymmetric, and no important publication bias was detected by Egger's tests (Egger's test $\mathrm{P}=0.055, \mathrm{P}=0.342$ and $\mathrm{P}=0.998$, respectively). Egger's test of total cholesterol suggested potential publication bias (Egger's test $\mathrm{P}=0.019$ ). The sensitivity analysis by the trim-and-fill method showed that the results was robust despite the potential bias (trim-and-fill method adjusted MD -1.14 mmol/L, 95\% Cl-1.57ख-0.72) (Figure S10-11 in appendix 3).

\section{Discussion}

\section{Findings and interpretations}

In this systematic review and meta-analysis of 67 randomized controlled trials, we found that Danhong injection adding to western medicine could significantly improve neurological function (e.g., improving NIHSS, Fugl-Meyer Assessment), self-care ability (e.g., improving Barthel index and ADL scores), hemodynamic outcomes (cerebral blood flow, average cerebral blood flow rate), blood lipids and Hcy in patients with ischemic stroke. Furthermore, patients with different age and receiving different baseline western medicines may have different benefits in hemodynamic status and neurological function, respectively.

NIHSS is a stroke-specific quantitative scale with excellent reliability and validity for ischemic stroke outcome, and measures strokerelated neurological deficits such as the level of consciousness, language function, visual field, eyes movement sensory function, and coordination[80-82]. In our study, we found that NIHSS scores were significantly decreased in patients receiving Danhong injection plus western medicine. This suggested that Danhong injection adding to western medicine would be beneficial in reducing the severity of ischemic stroke and improving neurological status. Overactivation of inflammatory factors and aggregation of platelets cause 
thrombosis and can lead to vascular occlusion, resulting in cerebrovascular ischemia, and then contribute to neurological deficit and physical dysfunction[83-85]. Since Danhong injection could inhibit platelet aggregation and boost the activity of fibrinogen dissolved, thus the add-on effects of Danhong injection and western medicine play a key role in improving NIHSS.

Fugl-Meyer Assessment is considered as one of the most comprehensive and evaluative measures for assessment of recovery in the poststroke patients[86]. As approximately $85 \%$ of patients with stroke present with arm weakness[87], our study found that the scores in Fugl-Meyer Assessment were significantly increased in patients treated by the integrated treatment with Danhong injection and western medicine, which suggests that Danhong injection adding to western medicine have the potential of enhancing limb function in stroke recovery.

PPAR-a, a transcription factor that regulates diverse aspects of lipid metabolism, plays a key role in the regulation of hepatic lipid metabolism[88, 89]. Our study showed that the combination of Danhong injection and western medicine had a noteworthy effect on reducing blood lipids (e.g., total cholesterol, triglycerides and LDL). Our study also showed that Danhong injection adding to western medicine performed a marked impact on increasing cerebral blood flow and average cerebral blood flow rate, which can be explained that Danhong injection could improve hemodynamic indices such as high shear viscosity, low shear viscosity, hematocrit, platelet aggregation rate[90].

Our study found the most common ADR in these reported RCTs were gastrointestinal reactions including nausea, flatulence and vomiting, which are a consequence of the drug's normal pharmacological effects[91]. About $58.2 \%$ of RCTs did not mention the information of ADRs in this study, thus we were unable to make a conclusion on safety so far. More credible evidences are warranted to confirm the safety of add-on effect of this drug in the future.

\section{Comparison with other studies}

Three previous systematic reviews and meta-analyses[9-11] have assessed the effect of Danhong injection for treatment of ischemic stroke. In terms of efficacy, all these three studies compared Danhong injection with other Chinese herbal injections such as Shuxuening injection, Yinxingdamo injection and so on. Instead of making a claim of comparative effectiveness between traditional Chinese medicines, we aimed to address whether the addition of traditional Chinese medicine to routinely used medications would improve the effects on ischemic stroke, which was a question that need strong evidence for conclusion. As it turned out, our study has provided reliable assessment of the effects.

\section{Strengths and Limitations}

Our study has several strengths. Firstly, we used rigorous methods to search, screen, and collect data from eligible RCTs. Secondly, we included several newly-published RCTs to summarize the efficacy and safety of Danhong injection in patients with ischemic stroke, aiming to provide a more comprehensive, up-to-date and new level of clinical evidence. Thirdly, we included a large number of RCTs, and used both patients reported important outcomes (e.g., NIHSS, Fugl-Meyer Assessment scores) and other objective outcomes (e.g., blood lipids, hemodynamic outcomes) to comprehensively assess the efficacy and safety of Danhong injection in patients with ischemic stroke. Fourthly, we conducted three prespecified subgroup analyses to explore sources of heterogeneity.

However, our study also has some limitations. The included RCTs may be at risk of high risk of bias. As a result, the findings may be susceptible to the bias. Additional well-designed and rigorously trials with long-term outcomes may be needed to further consolidate our findings.

\section{Conclusions}

Danhong injection adding to western medicine may improve the effects of neurological function, self-care ability, hemodynamic status, blood lipids and Hcy for patients with ischemic stroke. Furthermore, patients with different age and receiving different baseline western medicines may have different benefits in hemodynamic status and neurological function, respectively.

\section{Abbreviations}

ADEs: adverse drug events

ADL: activities of daily living 
ADRs: adverse drug reactions

Cls: confidence intervals

CNKI: China National Knowledge Infrastructure Database

Hcy: homocysteine

HDL: high-density lipoprotein

IMT: intima-media thickness

LDL: low-density lipoprotein

MD: mean difference

NIHSS: National Institutes of Health Stroke Scale Score

NR: not reported

PRISMA: Preferred Reporting Items for Systematic Reviews and Meta-analyses

RCTs: randomized clinical trials.

\section{Declarations}

\section{Ethics approval and consent to participate}

Not applicable; this systematic review utilizing publicly available information, does not require ethics approval.

\section{Consent for publication}

Not applicable.

\section{Availability of data and materials}

All data generated or analyzed during this study are supported by the published articles in database, and all data are included in this published article.

\section{Competing interests}

The authors declare that they have no competing interests.

\section{Funding}

This study was supported by National Key R\&D Program of China (Grant No. 2019YFC170 9804 and 2017YFC1700406), National Natural Science Foundation of China (Grant No. 719 04134), Sichuan Youth Science and Technology Innovation Research Team (Grant No. 2020 JDTD0015), China Center for Evidence Based Traditional Chinese Medicine (Grant No. 2020YJSZX-3) and 1.3.5 project for disciplines of excellence, West China Hospital, Sichuan University (Grant No. ZYYC08003).

\section{Authors' contributions}

Yu Ma: Literature searches, Data extraction, Data analysis, Writing- Original Draft. Ke Deng: Literature searches, Data extraction, Writing- Review \& Editing. Jiali Liu: Writing- Review \& Editing. Bin Ma: Writing- Review \& Editing. Fan Mei: Writing- Review \& Editing. Wen Hui: Writing- Review \& Editing. Xiaochao Luo: Writing- Review \& Editing. Minghong Yao: Writing- Review \& Editing. Yanmei Liu: Writing- Review \& Editing. Xuan Qin: Writing- Review \& Editing. Xu Zhou: Writing- Review \& Editing. Kang Zou: Writing- Review \& Editing. Ling Li: Conceptualization, Supervision, Project administration, Data analysis, Writing- Review \& Editing. Xin Sun: Conceptualization, Supervision, Project administration, Data analysis, Writing- Review \& Editing. All authors read and approved the final manuscript. 
Not applicable.

\section{References}

1. Collaborators GBDS: Global, regional, and national burden of stroke, 1990-2016: a systematic analysis for the Global Burden of Disease Study 2016. Lancet Neurol 2019, 18(5):439-458.

2. Powers WJ, Rabinstein AA, Ackerson T, Adeoye OM, Bambakidis NC, Becker K, Biller J, Brown M, Demaerschalk BM, Hoh B et al: Guidelines for the Early Management of Patients With Acute Ischemic Stroke: 2019 Update to the 2018 Guidelines for the Early Management of Acute Ischemic Stroke: A Guideline for Healthcare Professionals From the American Heart Association/American Stroke Association. Stroke 2019, 50(12):e344-e418.

3. van der Meij A, Wermer MJH: Vagus nerve stimulation: a potential new treatment for ischaemic stroke. The Lancet 2021, 397(10284):1520-1521.

4. Feng X, Li Y, Wang Y, Li L, Little PJ, Xu SW, Liu S: Danhong injection in cardiovascular and cerebrovascular diseases: Pharmacological actions, molecular mechanisms, and therapeutic potential. Pharmacol Res 2019, 139:62-75.

5. Xu W, Zhang Y, Yu Y, Li B, Liu J, Wang P, Wu H, Liu Q, Wei Z, Xiao H et al: Dose-dependent target diversion of Danhong injection on the Glu-GLT-1/Gly-GlyRa dynamic balance module of cerebral ischemia. Pharmacol Res 2018, 135:80-88.

6. Liu Y, Chen X, Xu P: Effect of Danhong Injection Combined with Edaravone in the Treatment of Acute Cerebral Infarction. Contemporary Medical Symposium 2019, 17(22):113-115.

7. Li Q, Hou S, Yang H, Su Y, Tu Z, Bi Q, Xiao W, Chen Y, Ye J, Pan D et al: The Effect of Dan Hong Injection Combined with Butylphthalide Injection on Acute Cerebral Infarction Patients Effects of BDNF, NPY and NSE. Chinese Journal of Integrative Medicine on Cardio/Cerebrovascular Disease 2020, 18(7):1148-1151.

8. Liu Q, Jin B: Effect of Danhong Injection Combined with Edaravone in the Treatment of Patients with Acute Cerebral Infarction and Its Influence on Cytokines, Cerebral Hemodynamics and Vascular Endothelial Function. Chinese Journal of Primary Medicine and Pharmacy 2020, 27(4):423-427.

9. Wang K, Zhang D, Wu J, Liu S, Zhang X, Zhang B: A comparative study of Danhong injection and Salvia miltiorrhiza injection in the treatment of cerebral infarction: A systematic review and meta-analysis. Medicine (Baltimore) 2017, 96(22):e7079.

10. Liu S, Wu JR, Zhang D, Wang KH, Zhang B, Zhang XM, Tan D, Duan XJ, Cui YY, Liu XK: Comparative efficacy of Chinese herbal injections for treating acute cerebral infarction: a network meta-analysis of randomized controlled trials. BMC Complement Altern Med 2018, 18(1):120.

11. Liu S, Wang K, Duan X, Wu J, Zhang D, Liu X, Zhao Y: Efficacy of Danshen Class Injection in the Treatment of Acute Cerebral Infarction: A Bayesian Network Meta-Analysis of Randomized Controlled Trials. Evid Based Complement Alternat Med 2019, 2019:5814749.

12. Page MJ, McKenzie JE, Bossuyt PM, Boutron I, Hoffmann TC, Mulrow CD, Shamseer L, Tetzlaff JM, Akl EA, Brennan SE et al: The PRISMA 2020 statement: an updated guideline for reporting systematic reviews. BMJ 2021, 372:n71.

13. Higgins JP, Altman DG, Gotzsche PC, Juni P, Moher D, Oxman AD, Savovic J, Schulz KF, Weeks L, Sterne JA et al: The Cochrane Collaboration's tool for assessing risk of bias in randomised trials. BMJ 2011, 343:d5928.

14. Egger M, Davey Smith G, Schneider M, Minder C: Bias in meta-analysis detected by a simple, graphical test. BMJ 1997, 315(7109):629-634.

15. Duval S, Tweedie R: Trim and fill: A simple funnel-plot-based method of testing and adjusting for publication bias in metaanalysis. Biometrics 2000, 56(2):455-463.

16. Cao S: Efficacy of Danhong Injection Combined with Alteplase in the Treatment of Acute Cerebral Infarction. Chinese Journal of Integrative Medicine on Cardio/Cerebrovascular Disease 2020, 18(5):827-830.

17. Cheng S: Effect of Danhong Injection Combined with Edaravone Injection in the Treatment of Senile Cerebral Infarction. China Modern Medicine 2019, 26(28):71-74.

18. Zhang Y: Efficacy and Safety Analysis of Danhong Injection Combined with Clopidogrel in the Treatment of Cerebral Infarction. Tianjin Pharmacy 2019, 31(2):46-47. 
19. Deng H: Danhong Injection Combined with Edaravone Injection on Clinical Efficacy Study of Patients with Cerebral Infarction. Clinical Research 2019, 27(1):101-103.

20. Li Y, Li Y, Zhang S, Wang H, Cai D: Clinical Efficacy of the Edaravone Combined with Danhong injection in the Treatment of Acute Cerebral Infarction and Its Influence on Serum Cytokine Level. Journal of Guangdong Medical University 2017, 35(5):469-471.

21. Li B: The Effect of Dan Hong injection Combined with Ozagrel Sodium on the Four Coagulation Items and Blood Lipids of Patients with Acute Cerebral Infarction. Modern Journal of Integrated Traditional Chinese and Western Medicine 2017, 26(1):49-51.

22. Wang L: Curative Effect Early Use of Danhong Injection Senile Cerebral Infarction. Journal of Shandong Medical College 2016, 38:258-260.

23. Zhang H: Efficacy of Vinpocefine Combined with Danhong Injection and Its Impact on Neurologic Function in Patients with Acute Cerebral Infarction. Internal Medicine of China 2015, 10(5):623-625.

24. Yin G: Clinical Study on Effect of Danhong Injection Combined with Hyperbaric Oxygen on Neural Cytokine of Cerebral Infarction Patients. Journal of Hunan University of Chinese Medicine 2014, 34(5):49-51.

25. Shen H: Efficacy of Danhong Injection Combined with Edaravone in the Treatment of Cerebral Infarction. Guide of China Medicine 2012, 10(24):580-581.

26. Zhuang M, Zhao X: Analysis of Danhong Injection in the Treatment of Type 2 Diabetes with Cerebral Infarction. Diabetes New World 2020:60-62.

27. Liu Y: Effects of Edaravone Combined with Danhong Injection on Diabetes Complicated with Cerebral Infarction. Clinical Medicine 2018, 38(8):84-87.

28. Shi J: Observation on the Clinical Efficacy of Danhong Injection Combined with Alprostadil in the Treatment of Acute Cerebral Infarction. Chinese Community Doctors 2018, 34(13):101-103.

29. Yang S, Yang M, Lei J, Yang X: Combined Treatment of Edaravone and Danhong Injection Ameliorates Brain Infarction by Regulating the Expression of Inflammation-related Factors. Immunological Journal 2018, 34(3):247-251.

30. Ma S: Dan Hong Injection Combined with Clopidogrel for the Treatment of Efficacy and Effect on Blood Rheology in Patients with Ischemic Stroke. Cardiovascular Disease Journal of Integrated Traditional Chinese and Western Medicine 2017, 5(24):100-101.

31. Liu W, Huang G: Clinical Efficacy of Fasudil Hydrochloride Combined with Danhong Injection in the Treatment of Acute Ischemic Cerebrovascular Disease. Strait Pharmaceutical Journal 2010, 22(6):158-160.

32. Chen Y: The Efficacy of Danhong Injection in the Treatment of Patients with Cerebral Infarction. Medical Frontier 2017, 7(13):6465.

33. Zhang M, Li M, Zheng Y, Tang L, Long S: Effect of Danhong Injection Combined with Alprostadil on Cerebral Vascular Reserve and Neurological Impairment in Patients with Ischemic Cerebral Infarction. Journal of North Sichuan Medical College 2018, 33(5):777780.

34. Zhang W: The Clinical Study of Danhong Injection Combined with Edaravone in the Treatment of Acute Ischemic Stroke. Chinese Journal of Primary Medicine and Pharmacy 2017, 24(7):1050-1053.

35. Pen Y: The Clinical Efficacy of Danhong Injection combined with Conventional Therapy for the Treatment of Diabetes Cmbined with Cerebral Ifarction in patients Clinical Research 2017(8):72.

36. Fan W, Huang X, Ma F, Xu M, Wan Q: Clinical Efficacy of Danhong Injection in the Treatment of Acute Cerebral Infarction. Progress in Modern Biomedicine 2018, 18(9):1772-1775.

37. Li Q, He Z, Li Q: Observation on Efficacy of Danhong Injection Combined with Clopidogrel in Treatment of Acute Cerebral Infarction. Evaluation and Analysis of Drug-use in Hospital of China 2017, 17(10):1336-1338.

38. Jiang P, Wang S, Guo C: The Treatment of Acute Cerebral Infarction with Danhong Injection. Journal of Henan Medical College 2020, 32(1):42-45.

39. Liu H: Effects of Danhong Injection on Cerebral Infarction in Patients with Type 2 diabetes Mellitus Effects of Vasodilatory Function and Endothelial tPA and NO Reserve Release. Chinese Journal of Integrative Medicine on Cardio/Cerebrovascular Disease 2017, 15(24):3177-3180.

40. Ge Y: Effectiveness of Dan Hong Injection Combined with Atorvastatin Calcium in the Treatment of Lacunar Cerebral Infarction Medical Journal of Chinese People's Health 2018, 30(19):62-64. 
41. Yun H, Li J, Hu F: Effect of Dan Hong Injection on Cerebral Hemodynamics and Hemorheology in Patients with Cerebral Infarction. Hainan Medical Journal 2017, 28(7):1059-1061.

42. Liu Q, Peng G, Lin H: Effect of Danhong Injection on TCM Syndrome Score, NIHSS Integral and Inflammatory Factor in Patients with Acute Cerebral Infarction. Chian Practical Medicine 2019, 14(16):130-131.

43. Dai J: Clinical Efficacy of Danhong Injection in Patients with Acute Cerebral Infarction and It's Influence on NIHSS and ADL Scores. Clinical Medicine 2018:22-23.

44. Yang J: Effect of Danhong Injection Combined with Atorvastatin Calcium on NIHSS Score and Ability of Daily Living in Lacunar Cerebral Infarction. Inner Mongolia Medical Journal 2018, 50(8):997-998.

45. Li J: An Investigation of Therapeutic Efect of Danhong Injection Combined with Edaravone for Treatment of Patients with Acute Cerebral Infarction. Chinese Journal of Integrated Traditional and Western Medicine in Intensive and Critical Care 2015, 22(2):178180.

46. Liu Z, Wang H, Liu L, Feng C, Liu Y, Liu X: Influence of Danhong Injection on Endothelial Progenitor Cells冈lnflammatory Factor and Neural Function in Patients with Acute Cerebral Infarction. International Journal of Laboratory Medicine 2017, 38(23):3265-3264.

47. Luo S: Treatment of Acute Ischemic Stroke with Danhong Injection Combined with Butylphthalide Soft Capsule for the Efficacy of Post-stroke and the Effect on Cognitive Function. Modern Practical Medicine 2018, 30(9):1171-1173.

48. Kang M, Wen C, Liu Y, Sun J, Zhang B: Effect of Danhong Injection and Tirofiban on Acute Cerebral Infarction and Associated NFKB Inflammation Signal Pathway. Chinese Journal of Rational Drug Use 2020, 5(17):56-60.

49. Yang J, Liu Q: Effect of Dan Hong Injection Combined with Clopidogrel on Coagulation, LPO, sICAM-1 and IGF-1 Levels in Patients with Acute Cerebral Infarction. Chinese Journal of Integrative Medicine on Cardio/Cerebrovascular Disease 2018, 16(8):11191122.

50. Wang N, Guo H, Liu P, Guo S, Yang Q: Levels of IL-8هFibulin-5 and P-selectin in Patients with Acute Cerebral Infarction and the Intervention Effect of Danhong Injection. Journal of Liaoning University of Traditional Chinese Medicine 2016, 18(11):177-179.

51. Yuan D: Clinical Efficacy of Danhong Combined with Edaravone Injection in the Treatment of Acute Cerebral Infarction and Its Influence on Serum Related Factors. Chinese and Foreign Medical Research 2019, 17(36):130-132.

52. Su X, Luo S, Long J: The Efficacy of Danhong Injection in Treating 38 Cases of Cerebral Infarction. Guangxi Medical Journal 2012 , 34(11):1543-1545.

53. Luo Y, Xu D, Xu X: 90 Cases of Acute Cerebral Infarction Treated with Danhong Injection Combined with Ozagrel. Chinese Journal of Primary Medicine and Pharmacy 2012, 19(20):3136-3137.

54. Zou L, Li M, Li H, Chen X: Clinical Study of Vincristine Combined with Danhong Injection in the Treatment of Acute Cerebral Infarction. Chinese Journal of Information on Traditional Chinese Medicine 2013, 20(12):67-68.

55. Liang Z, Zhang H, Qi D, Wen M: Effect of Dan Hong Injection on Serum of Acute Ischemic Stroke Patients Inflammatory Factors in Acute Ischemic Stroke Patients. Journal of Emergency in Traditional Chinese Medicine 2014, 23(11):2115-2116.

56. Ma J, Yu N, Wu H, Chen M, Ren Z, Zhao D: Effect of Danhong Injection on Nerve Function and Hemorheology for Patients with Acute Ischemic Stroke. Chinese Journal of Experimental Traditional Medical Formulae 2015, 21(20):204-207.

57. Fan Z, Chen H, Jiang C: Clinical Eficacy of Danhong Injection on Cerebral Ischemic Stroke and Its Influence on Blood Lipid Metabolism and Nerve Functio. Chinese Journal of Evidenced Based Cardiovasc Medicine 2015, 7(6):809-811.

58. Zeng Y, Liao J, Chen D: Dan Red Injection Adr Mentioned Joint Effects of Neural Function in Patients with Cerebral Infarction Injection Treatment Research. Drugs and Clinic 2016, 13(17):45-52.

59. Feng Q, Xu M, Zhang W, Huang J, Liu T, Huangpu L, Zheng M, Tan L: Clinical Study on Intra-arterial Thrombolysis with Rt-PA and Dan Red Injection for Acute Cerebral Infarction. Journal of Emergency in Traditional Chinese Medicine 2016, 25(2):239-241.

60. Ou Y, Liu Q: Clinical Efect of Danhong Injection in Treating Acute Cerebral Infarction. Journal of Modern Health 2017, 33(11):16011603.

61. Wu L, Bao D: Clinical Observation on the Adjuvant Treatment of Acute Cerebral Infarction with Danhong Injection. Journal of New Chinese Medicine 2016, 48(4):20-22.

62. Chen Y, Shi X: Clinical Effect of Danhong Injection Combined with Rehabilitation Exercise on Acute Cerebral Infarction and Its Effect on Motor Function. Chinese Journal of Biochemical Pharmaceutics 2017, 10(37):147-149. 
63. Qiu C, Zang Y, Wang Q, Wang C, Zhao C: Effect of Danhong Injection in the Treatment of Senile Acute Cerebral Infarction and Its Effect on Blood Lipid冈Blood Rheology and High Sensitivity C-reactive Protein. Modern Medical Journal 2017, 45(1):71-74.

64. Liu F, Li Y, Liu G, Wang W, Zhang Q: Effects of Danhong Injection on the Serum Blood Uric Acid and Bilirubin Levels of Patients with Acute Cerebral Infarction. Progress in Modern Biomedicine 2017, 17(7):1321-1323.

65. Wei W, Wang S, Li N: The Effect of Danhong Injection on the Improvement of Nerve Function and Hemorheology in Patients with Acute Cerebral Infarction. Medical Innovation of China 2017, 14(36):27-30.

66. Jing W, Yu J: Study on the Clinical Efficacy of Danhong Injection in the Adjuvant Treatment of Acute Cerebral Infarction. Chian Practical Medicine 2020, 12(2):138-139.

67. Li X, Huang S, Yu T, Lan X: Efficacy of Danhong Injection Combined with Atorvastatin Calcium for Diabetes Mellitus Complicated with Cerebral Infarction. Guangxi Medical Journal 2018, 40(9):1009-1012.

68. Jin Y, Jin L, Liao Y, Liu F: Dan Hong Injection Combined with Recombinant Tissuetype Fibrinogen Activator on the Neurological Coagulation Function of Patients with Acute Cerebral Infarction and Vascular Endothelial Function Level in Patients with Acute Cerebral Infarction. Chinese Remedies \& Clinics 2019, 19(19):3400-3402.

69. Chai Y, Zhang Y, Shi Y, Kang L, Zhu Z: Efficacy of the Combination of Ozagrel Sodium, Danhong Injection and Aspirin (ASA) on Acute Cerebral Infarction and the Effect on Patients' ADL Scores. Guizhou Medical Journal 2019, 43(12):1922-1924.

70. Chen H, Zhang F, Wang W, Lv R: Efficacy of Dan Hong Injection in the Adjuvant Treatment of Acute Cerebral Infarction and Its Effect on Oxidative Stress. Modern Journal of Integrated Traditional Chinese and Western Medicine 2019, 28(18):1986-1988.

71. Li J, Tian Y: Effect of Dan Hong Injection Combined with Eurefrin in the Treatment of Acute Ischemic Stroke. Henan Medical Research 2019, 28(14):2630-2631.

72. Zhu J, Qi J: Effect of Danhong Injection in the Treatment of Acute Cerebral Infarction and Its Effect on Patients' Neurological Function and Inflammatory Factor Levels. Clinical Medicine 2020, 40(2):95-97.

73. Cao Y, Huang H, Duan D, Song Y, Wang Y: Clinical Efficacy and Safety Analysis of Danhong Injection in Treating Acute Cerebral Infarction. HEBEI MEDICINE 2019, 25(4):676-679.

74. Song L, Yang M: The Application Value in Dan Hong Injection Combined with Conventional Treatment in Patients with Acute Cerebral Infarction Modern Journal of Integrated Traditional Chinese and Western Medicine 2014, 23(14):1522-1524.

75. Lv Q, Shi F, Shan K, Jing J: The Effect of Recombinant Tissue Plasminogen Activator Combined with Danhong Injection on Seruln Cytokines and Inflammatory Factor Levels in Patients with Acute Cerebral Infarction. Stroke and Nervous Diseases 2018, 25(6):635-639.

76. Huo S, Guo Y, Li J: The Effect of Danhong Injection Combined with Calf Serum Deprotein Injection for the Treatment of Elderly Acute Cerebral Infarction. Clinical Medicine 2020, 40(2):104-105.

77. Cao F, Mei B: The Clinical Effect of Danhong Injection in the Treatment of Branch Atheromatous Disease Stroke and Nervous Diseases 2016, 23(6):442-445.

78. Ren J, Zhang A, Cao F: Effect of Dan Hong Injection on Plasma IL-6 and TNF-a Concentrations in ACI Patients. Journal of Radioimmunology 2011, 24(5):570-571.

79. Xue W, Li L: Effection of Danhong Injection on Atherosclerotic Plaque in Carotid Artery of Ischemic Stroke. Medical Journal of Chinese People's Health 2010, 22(6):644-645.

80. Boone M, Chillon JM, Garcia PY, Canaple S, Lamy C, Godefroy O, Bugnicourt JM: NIHSS and acute complications after anterior and posterior circulation strokes. Therapeutics and Clinical Risk Management 2012, 8:87-93.

81. Scott E. Kasner, Julio A. Chalela, Jean M. Luciano, Brett L. Cucchiara, Eric C. Raps, Michael L. McGarvey, Molly B. Conroy, Localio AR: Reliability and validity of estimating the NIH stroke scale score from medical records. Stroke 1999, 30(8):1534-1537.

82. Muir KW, Weir CJ, Murray GD, Povey C, Lees KR: Comparison of neurological scales and scoring systems for acute stroke prognosis. stroke 1996, 27(10):1817-1820.

83. Komurcu HF, Gozke E, Dogan Ak P, Kalyoncu Aslan I, Salt I, Ozgenc Bi Er Cl: Changes in neutrophil, lymphocyte, platelet ratios and their relationship with NIHSS after rtPA and/or thrombectomy in ischemic stroke. J Stroke Cerebrovasc Dis 2020, 29(8):105004

84. Franks ZG, Campbell RA, Weyrich AS, Rondina MT: Platelet-leukocyte interactions link inflammatory and thromboembolic events in ischemic stroke. Ann N Y Acad Sci 2010, 1207:11-17. 
85. Xu XR, Carrim N, Neves MA, McKeown T, Stratton TW, Coelho RM, Lei X, Chen P, Xu J, Dai X et al: Platelets and platelet adhesion molecules: novel mechanisms of thrombosis and anti-thrombotic therapies. Thromb J 2016, 14(Suppl 1):29.

86. Gladstone DJ, Danells CJ, Black SE: The fugl-meyer assessment of motor recovery after stroke: a critical review of its measurement properties. Neurorehabil 2002, 16:232-240.

87. Dawson J, Pierce D, Dixit A, Kimberley TJ, Robertson M, Tarver B, Hilmi O, McLean J, Forbes K, Kilgard MP et al: Safety, Feasibility, and Efficacy of Vagus Nerve Stimulation Paired With Upper-Limb Rehabilitation After Ischemic Stroke. Stroke 2016, 47(1):143-150.

88. Kersten S, Seydoux J, Peters JM, Gonzalez FJ, Desvergne B, Wahli W: Peroxisome proliferator-activated receptor alpha mediates the adaptive response to fasting. The Journal of clinical investigation 1999, 103(11):1489-1498.

89. Li AC, Glass CK: PPAR- and LXR-dependent pathways controlling lipid metabolism and the development of atherosclerosis. J Lipid Res 2004, 45(12):2161-2173.

90. Jiang Y, Lian YJ: Effects of Danhong injection on hemodynamics and the inflammation-related NF-kappaB signaling pathway in patients with acute cerebral infarction. Genet Mol Res 2015, 14(4):16929-16937.

91. Li XL, Tang JF, Li WX, Li CX, Zhao T, Zhao BC, Wang Y, Zhang H, Chen XF, Xu T et al: Postmarketing Safety Surveillance and Reevaluation of Danhong Injection: Clinical Study of 30888 Cases. Evid Based Complement Alternat Med 2015, 2015:610846.

\section{Figures}
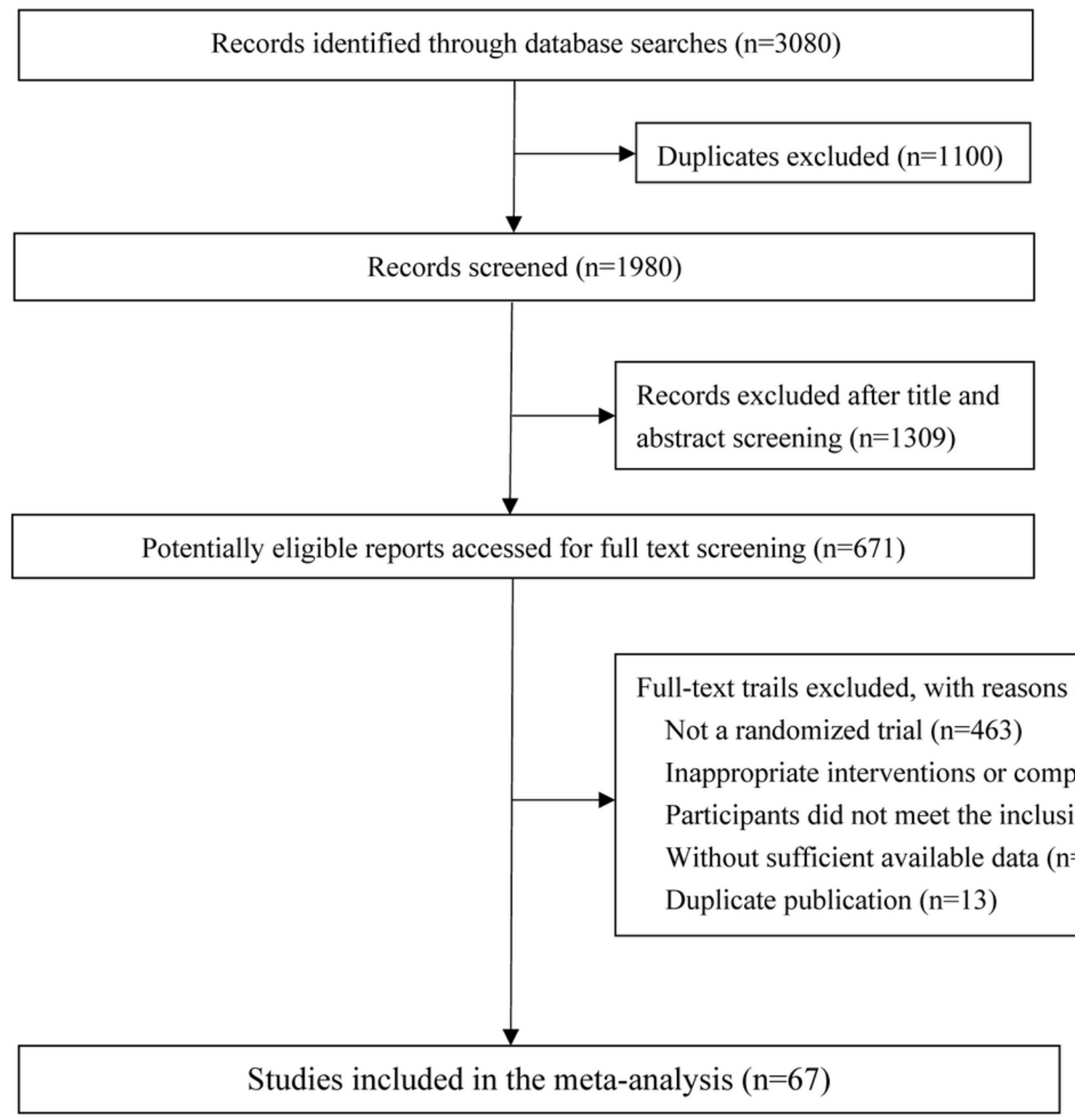


\section{Figure 1}

Flow chart of study selection

Figure 2

Meta-analysis of NIHSS between DHI+WM and WM in patients with Ischemic Stroke

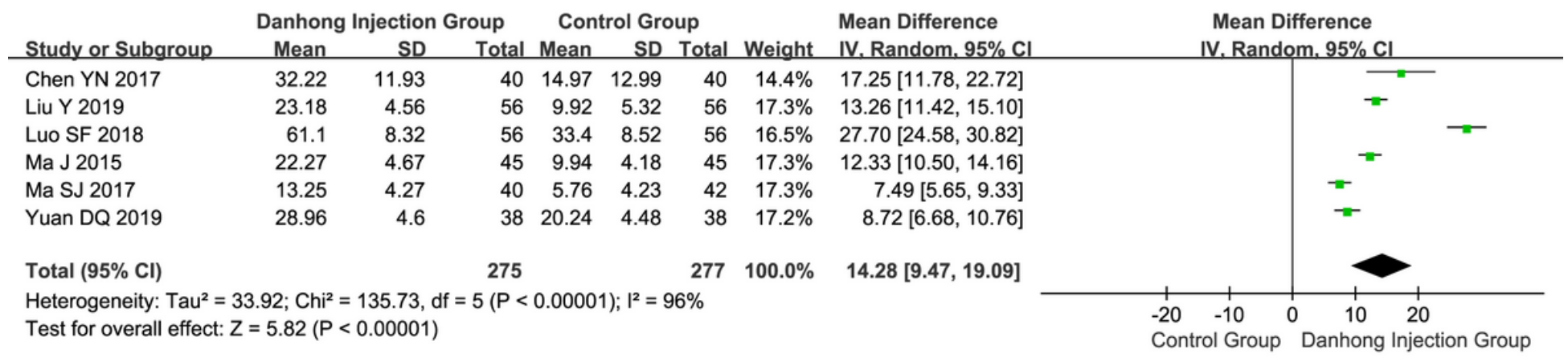

\section{Figure 3}

Meta-analysis of Fugl-Meyer Assessment between DHI+WM and WM in patients with Ischemic Stroke

\section{Figure 4}

Meta-analysis of total cholesterol between DHI+WM and WM in patients with Ischemic Stroke

\section{Figure 5}

Meta-analysis of Hcy between DHI+WM and WM in patients with Ischemic Stroke

\section{Supplementary Files}

This is a list of supplementary files associated with this preprint. Click to download.

- Appendix1Searchstrategies.docx

- Appendix2Supplementaryfigures.docx

- Appendix3Supplementarytables.docx 